\title{
Grafos de Aplicações Estáveis entre Superfícies Fechadas
}

\author{
Catarina M. J. Sánchez, \\ Depto de Matemática, UFV, \\ 36570-900, Viçosa, MG \\ E-mail: cmendes@ufv.br
}

\begin{abstract}
Resumo: Neste trabalho veremos que todo grafo com pesos nos vértices pode ser associado a alguma aplicação estável entre duas superfícies fechadas e orientadas, generalizado resultados de aplicações estáveis de superfícies fechada e orientadas na esfera.
\end{abstract}

Palavras-chave: Aplicações Estáveis, Grafos com Pesos, Superfícies Fechadas

\section{Introdução}

As aplicações estáveis entre duas superfícies fechadas e orientadas, localmente podem ser vistas como aplicações do plano no plano. O estudo de aplicações do plano no plano foi introduzido em 1955 por Whitney [10], onde determinou que um germe (pequena vizinhança do ponto) de aplicação em cada ponto é equivalente a um ponto regular ou ponto singular de dobra ou uma cúspide. Whitney também determinou que o conjunto de aplicações estáveis entre duas superfícies formam um subconjunto aberto e denso no espaço de todas as aplicações suaves.

O conjunto singular $\Sigma f$ de uma aplicação estável $f$ entre duas superfícies fechadas e orientadas $M$ e $N$ consiste em uma coleção de curvas fechadas, disjuntas e mergulhadas sobre $M$ e as componentes do conjunto regular, complementar de $\Sigma f$ em $M$, consiste em uma coleção de superfícies orientadas que tem como bordo as curvas singulares. A imagem de $\Sigma f$, conhecido como contorno aparente, consiste em uma coleção de curvas fechadas e imersas sobre $N$, com possíveis interseções transversais e singularidades correspondentes às cúspides, sendo ambas em número finito.

Em [4] e [1], foi introduzido grafos com pesos nos vértices associados às aplicações estáveis de superfícies fechadas e orientadas no plano, chamado de grafo dual de aplicações estáveis. Estes grafos classificam por completo o par $(M, \Sigma f)$ e foram estudados como invariantes topológicos de aplicações estáveis no plano em [2], de aplicações estáveis na esfera em [3] e no plano projetivo $(\mathrm{em}[6]$.

O grafo de aplicações estáveis é uma ferramenta muito útil na construção de exemplos destas aplicações entre variedades e também foi estendido para aplicações de Gauss estáveis, de superfícies fechadas e orientadas imersas no 3-espaço, em [7] e para aplicações estáveis de três variedades fechadas e orientadas no 3-espaço em [8].

Na teoria de singularidades de aplicações diferenciáveis, em geral, são utilizadas ideias e técnicas das áreas de Geometria Diferencial, Análise e Álgebra para estudar o comportamento (local, semi-global e global) das singularidades, enquanto que no estudo de grafos associados às aplicações estáveis entre superfícies, em geral, as técnicas estão mais relacionados à Topologia como, por exemplo, a própria construção do grafo, que é um invariante topológico, a relação da característica de Euler entre o grafo e a superfície domínio e as cirurgias de aplicações estáveis (introduzida em [2]).

O principal resultado deste trabalho generaliza o caso das aplicações estáveis de superfícies orientadas sobre a esfera em [?] cujo enunciado é:

Teorema 4.5 Todo grafo com pesos nos vértices pode ser associado a alguma aplicação, com grau máximo $T / n$, de uma superfície fechada e orientada $M$ com gênro $T$ sobre uma superfície fechada e orientada com gênero $n$. 


\section{Grafos associado a aplicações estáveis entre superfícies}

Denotamos por $C^{\infty}(M, N)$ o espaço de todas as aplicações de classe $C^{\infty}$ de $M$ em $N$. Duas aplicações $f, g \in C^{\infty}(M, N)$, são ditas $\mathcal{A}$-equivalente quando existem difeomorfismos $\phi: M \rightarrow$ $N$ e $\psi: M \rightarrow N$ tais que $g=\psi \circ f \circ \phi^{-1}$. Uma aplicação $f \in C^{\infty}(M, N)$, é dita estável se para todas aplicações suficientemente perto de $f$ (na $C^{\infty}$-topologia de Whitney) são equivalentes a $f$. Segundo Whitney [10], o conjunto de todas aplicações estáveis, que denotaremos por $\mathcal{E}(M, N)$, é aberto e denso no espaço das aplicações suaves $C^{\infty}(M, N)$.

A Figura 1 ilustra três diferentes aplicações do toro na esfera: a primeira e a segunda (da esquerda para direita) tem grau 1 e a terceiera tem grau 2.
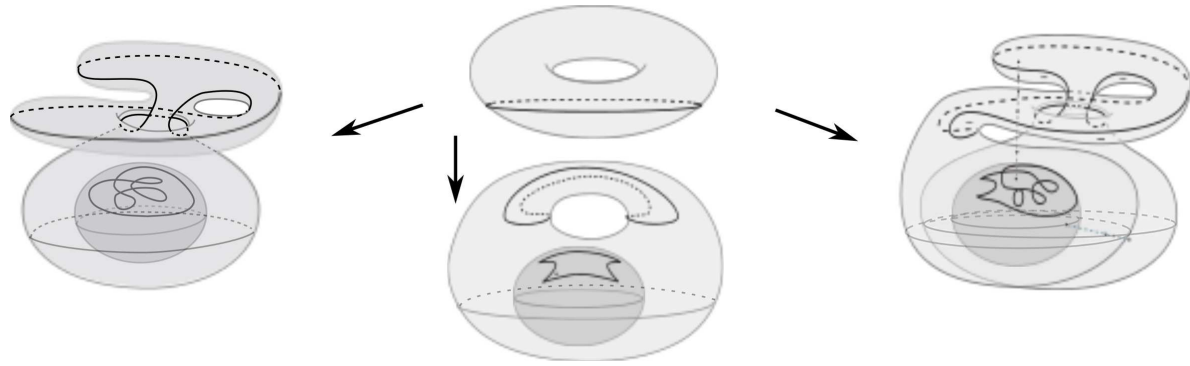

Figura 1: Exemplos de aplicações do toro na esfera.

Dada uma aplicação estável $f \in \mathcal{E}(M, N)$, um ponto $x$ em $M$ é dito ponto regular de $f$ se a aplicação $f$ é um difeomorfismo local na vizinhança do ponto $x$, caso contrário dizemos que $x$ é um ponto singular. As singularidades locais de $f$, segundo um Teorema de Whitney [10], são do tipo dobra (com forma local $\left.(x, y) \mapsto\left(x^{2} ; y\right)\right)$ e do tipo cúspides (com forma local $\left.(x, y) \mapsto\left(x^{3}+y x, y\right)\right)$.

O conjunto de todos os pontos sigulares de $f$, denotado por $\Sigma f$, é chamado de conjunto singular de $f$ e está formado por um conjunto de curvas simples, disjuntas e mergulhadas em $M$ (subvariedade de codimensão $1 \mathrm{em} M$ ). O conjunto de todos os pontos não sigulares de $f$, denotado por $M \backslash \Sigma f$, é chamado de conjunto regular e consiste em um número finito de regiões de $M$ que são imersas em $N$ por $f$. As regiões regulares tem como bordo as curvas de $\Sigma f$. A imagem destas curvas singulares, denotado por $B f=f(\Sigma f)$, é chamado de contorno aparente de $f$ (ou conjunto de ramificação) e está formado por curvas suaves em $N$ com um número finito de pontos duplos, de interseções trasversas, e possíveis pontos de cúspides.

Grafos de aplicações estáveis. Dadas duas superfícies fechadas e orientadas $M$ e $N$ duas uma aplicação estável $f: M \longrightarrow N$, as curvas conjunto singular $\Sigma f$ separam as regiões do conjunto regular $M \backslash \Sigma f$ em componentes conexas disjuntas. Então podemos associar ao par $(M, \Sigma f)$ um grafo $\mathcal{G}$ com pesos nos vértices da seguinte forma:

i) cada região regular $U$ de $M \backslash \Sigma f$ fazemos corresponder a um vértice $v$ em $\mathcal{G}$,

ii) cada curva $\alpha$ de $\Sigma f$ fazemos corresponder uma aresta $a$ em $\mathcal{G}$,

iii) um vértice $v$ recebe o peso $t$ se a região regular correspondente a $v$ tem gênero $t$ (soma de $t$ toros).

iv) Uma aresta $a$ conecta o vértice $v$ se, e somente se, a curva singular correspondente a $a$ está no bordo da região regular correspondente a $v$.

Definição 2.1. O grafo $\mathcal{G}$ associado a $f \in \mathcal{E}(M, N)$ é chamado de grafo dual de $f$.

Definição 2.2. Seja $\mathcal{G}$ um grafo com $E$ arestas, $V$ vértices e pesos $t_{i}$ nos vértices $v_{i}$, para $i=1, \cdots, V$. Chamaremos de característica de $\mathcal{G}$ o vetor $C(\mathcal{G})=(V, E, T)$, onde $T=\sum_{i=1}^{V} t_{i}$. 
Em [1] foi estendida a definição de grafos com pesos nos vértices para o par $(M, \mathcal{C})$, onde $\mathcal{C}$ é um conjunto de curvas fechadas simples e disjuntas sobre a superfície orientada $M$, e foi provado que todo grafo com pesos nos vértces está associado a algum par $(M, \mathcal{C})$, onde o gênero de $M$ é dado por $g(M)=1-V+E+T$.

Proposição 2.3. Seja $\mathcal{G}$ um grafo com $C(\mathcal{G})=(V, E, T)$ associada a um par $(M, \mathcal{C})$, onde $\mathcal{C}$ é um conjunto de curvas simples, fechadas e disjuntas sobre a superfície fechada e orientada $M$. Então a característica de Euler de $M$ é dado por $\chi((M)=2(V-E-T)$.

Demonstração. A característica de Euler de cada componente $M_{i}$ de $M \backslash \mathcal{C}$ com gênero $t_{i}$ e $E_{i}$ componentes de bordos de $M_{i}$, sabemos da Topologia Algébrica que, é dada por $\chi\left(\left(M_{i}\right)=\right.$ $2-2 t_{i}-E_{i}$. A interseção entre duas componentes regulares quaisquer é um curva fechada e tem a característica de Euler igual a zero. $\operatorname{Logo} \chi(M)=\sum_{i=1}^{V} \chi\left(M_{i}\right)=\sum_{i=1}^{V}\left(2-2 t_{i}-E_{i}\right)=$ $2(V-T-E)$, pois cada componente singular $E_{i}$ é bordo de duas componente regulares.

Uma região de $M \backslash \Sigma f$ será dita positiva se tem a orientação preservada por $f$ e negativa no caso contrário.

Observe que se $M$ é orientada, toda curva do conjunto singular de uma aplicação $f \in \mathcal{E}(M, N)$ separa uma região regular positiva de uma região regular negativa. Ou seja, o conjunto singular cria uma bipartição no conjunto regular, consequentemente, podemos afirmar que todo grafo associado a uma aplicação estável de uma superfície orientada $M$ sobre uma superfície $N$ é bipartido.

Uma pergunta natural é: Quais grafos bipartidos com pesos inteiros positivos nos vértices podem ser associados a aplicações estáveis entre superfícies fechadas e orientadas?

Dada uma aplicação $f \in \mathcal{E}(M, N)$ com grau $d$, denotaremos por $M^{+}$e $M^{-}$, respectivamente, o conjunto de todas as regiões regulares positivas e negativas, por $V^{ \pm}$o número de componentes regulares de $M^{ \pm}$e por $T^{ \pm}$a soma total dos gêneros das componentes de $M^{ \pm}$. Aplicando cirurgias de aplicações estáveis e transições de codimensão um, no espaço das aplicações suaves, foi provado em [5] que $\left(V^{+}-V^{-}\right)-\left(T^{+}-T^{-}\right)=d\left(1-g(N)-\left(C^{+}-C^{-}\right)\right.$, onde $\left(C^{+}-C^{-}\right)$ corresponde a diferença dos números de cúspides que apontam, respectivamente, para $M^{+}$e $M^{-}$. O próximo resultado é uma consequência imediata.

Teorema 2.4. Todo grafo associado a uma aplicação estável sem cúspides $f \in \mathcal{E}(M, N)$ com grau d satisfaz $\left(V^{+}-V^{-}\right)-\left(T^{+}-T^{-}\right)=d(1-g(N)$.

Em [3] foi provado que todo grafo com pesos nos vértices pode ser realizado por uma aplicação sem cúspides na esfera com grau $d=\left(V^{+}-V^{-}\right)-\left(T^{+}-T^{-}\right)$.

\section{Cirurgia de Aplicações estáveis}

Nosso objetivo é mostrar que grafos bipartidos com pesos nos vértices podem ser associados a aplicações estáveis entre superfície orientadas. Uma forma de construir estas aplicações é através de cirurgias de aplicações estáveis.
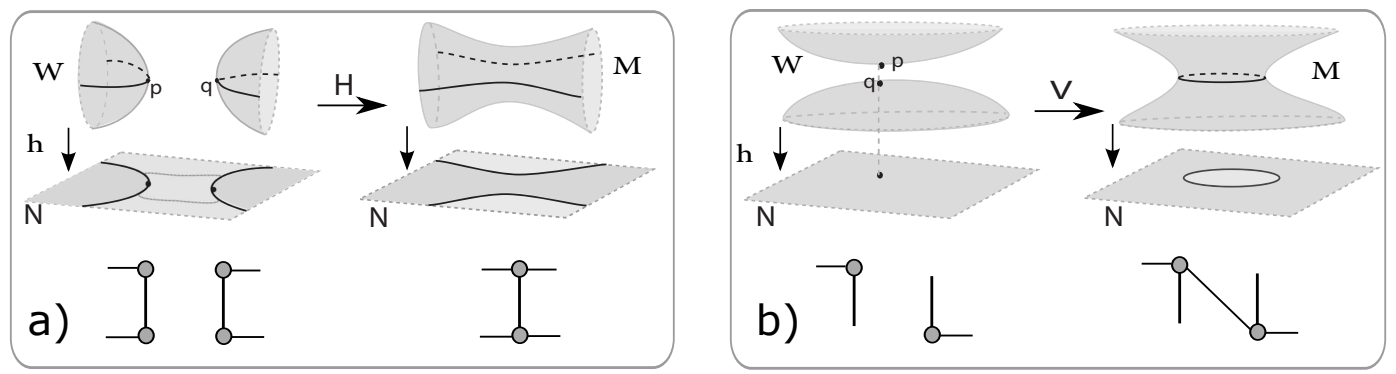

Figura 2: $H$ : cirurgia horizontal e $V$ : cirurgia vertical 
Em [2] foi definido dois tipos de cirurgias de aplicações estáveis entre superfícies, chamadas de cirurgia horizontal e cirurgia vertical (respect.) ilustrada na Figura 2. Nas duas cirurgias um par de discos disjuntos da superfície é removido e substituido por um tubo conectando os bordos, onde estendemos a aplicação sobre o interior do tubo, como veremos a seguir:

a) Cirurgia Horizontal $H$. Dada uma aplicação estável $h$ entre duas superfícies $W$ e $N$, uma ponte é um retângulo $\beta$ com o interior mergulhado no complementar do contorno aparente $B h$ em $N$ e com dois lados opostos sobre intervalos $h(I)$ e $h(J)$, de forma compatível com a orientação de $B h$ (ver a) Figura 2 ). A aplicação estável $h_{\beta}$ (que depende da escolha do lugar da ponte $\beta$ ) é construida como segue: Um ponte $\beta$ encontra $h(W)$ em dois intervaloss $h(I)$ e $h(J)$ contidos em $B h$. Escolha pequeno discos em $W$ um conectando $I$ e outro $J$. Repasse a seus interiores por um tubo respeitando a orientação de $W$ de forma a obter uma orientação na superfície $M$. A aplicação $h$, como ilustrada na Figura 2(a), pode ser estendida sobre o tubo de forma a obter a aplicação estável $h_{\beta}$.

Em particular, se $W$ é a união disjunta das superfícies $P$ e $Q$, denotamos por $W=P \sqcup Q$, onde $p$ e $q$ denota a restrição da aplicação $h$ a $P$ e a $Q$; com $I$ em $P$ e $J$ em $Q$. Neste caso, obtemos a soma horizontal $p \oplus_{H} q: M \longrightarrow N$. Em outras palavras $h=p \cup q$ e $(f \cup l)_{\beta}=p \oplus_{H} q$.

b) Cirurgia Vertical $V$. Neste caso, fazemos a soma conexa pela identificação de dois pequenos discos no domínio sem pontos singulares, um contindo numa componente regular positiva e outro numa componente regular negativa (ver b) Figura 2) cuja imagem em $N$ coincide.

Os discos são substituídos por um tubo que é aplicado em $N$, com uma curva singular ao redor no meio do tubo. Assim a cirurgia adiciona uma curva disjunta mergulhada no conjunto de ramificação. Denotamos esta soma como $p \oplus_{V} q$.

A Figura 3 ilustra exemplos de cirurgias horizontais e verticais de uma aplicação estável sobre a esfera, onde $M$. Da esquerda para a direita, a primeira figura corresponde a uma aplicação da esfera na esfera com cinco curvas singulares e dois pontos de cúspides cada uma, a segunda figura corresponde a uma aplicação do 4-toro na esfera, que pode ser obtida por quatro cirurgias horizontais sobre sobre a primeira aplicação e a terceira (os "olhos do morcego") pode ser obtido por duas cirurgias vérticais sobre a segunda.
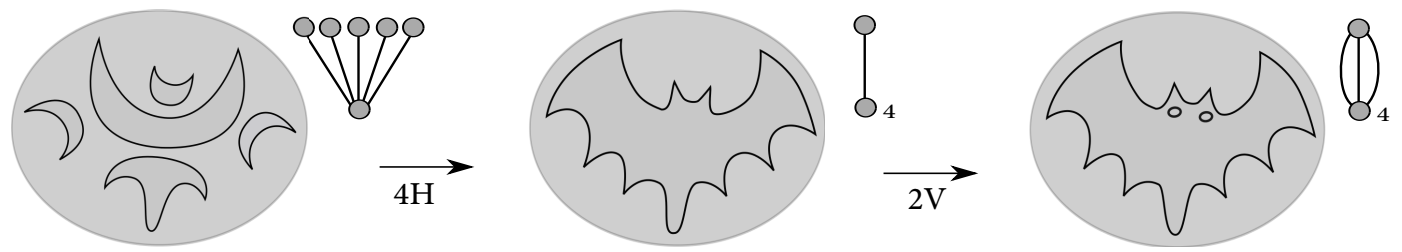

Figura 3: Exemplos de cirurgias sobre uma superfície conexa.

\section{Realização de grafos bipartidos}

Lembrando que se $f: M \longrightarrow N$ é uma aplicação estável associada a um grafo $\mathcal{G}$, com $C(\mathcal{G})=$ $(V, E, T)$, então $\mathcal{G}$ é bipartido e a superfície orientada $M$ tem gênero $g(M)=1-V+E+T$.

Observe que que sempre podemos obter uma aplicação de grau zero $g: M \longrightarrow N$, onde $M$ é uma superfície, pela composição de uma aplicação $h: M \longrightarrow \mathbb{R}^{2}$ com um mergulho da imagem de $h$ sobre $N$ que é a aplicação $j: h(M) \subset \mathbb{R}^{2} \longrightarrow N$. Toda aplicação com grau zero sobre $N$ é homotópica a alguma aplicação formada por esta composição e será chamada de aplicação planar.

Se $N$ é uma supefície fechada e orientada com gênero $n \neq 1$, qualquer aplicação com conjunto singular vazio sobre $N$ é estavelmente homotópica à identidade $I d: N \longrightarrow N$ e tem grau um, enquanto que para o toro $T^{2}$, superfície com gênero um, existem aplicações $l: T^{2} \longrightarrow T^{2}$ com grau $d>1$ e com o conjunto singular vazio. 
Veremos a seguir como realizar grafos bipartidos, com diferentes pesos, número de vértices e arestas, sobre uma superfície $N$. Mas antes veremos alguns resultados, provados em [1] e [2], sobre grafos de aplicações estáveis de superfícies fechadas e orientadas no plano.

Observe que toda árvore com $V$ vértices pode ser decomposta em subgrafos $A_{1}, \cdots, A_{k}$, $k<V$, onde todos os subgrafos tem no máximo quatro vértices. Este grafos podem ser realizados por aplicações $h_{i}: S^{2} \longrightarrow \mathbb{R}^{2}, i=1, \cdots, k$, como ilustra a Figura 4 .
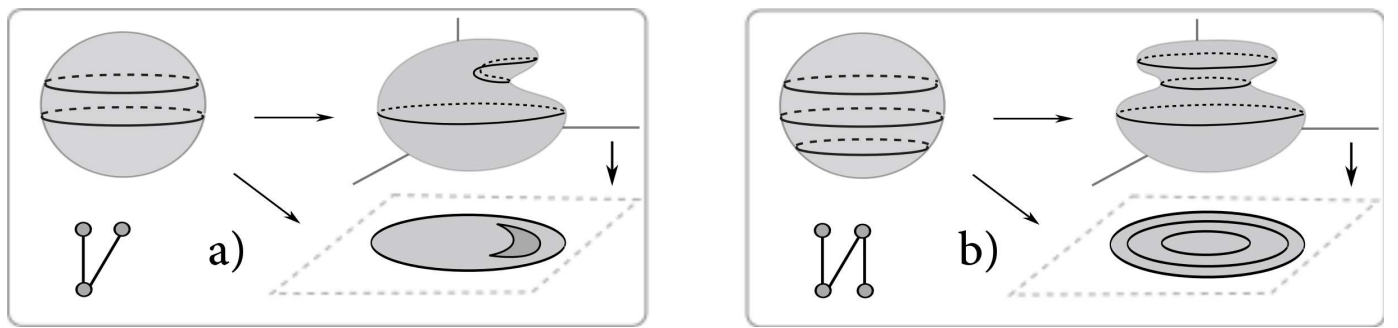

Figura 4: Aplicações da esfera no plano, com duas e três curvas singulares.

Uma aplicação $h: S^{2} \longrightarrow \mathbb{R}^{2}$ que realiza $A$ pode ser obtida por $k-1$ cirurgias horizontais entre as $k$ aplicações $h_{i}, i=1, \cdots, k$, respeitando a decomposição de $A$. Com isto podemos afirmar o próximo resultado.

Teorema 4.1. Toda árvore $A$ com $C(A)=(V, V-1,0)$ pode ser realizada por alguma aplicação $k: S^{2} \longrightarrow \mathbb{R}^{2}$.

O grafo com única aresta correspondente a uma aplicação estável com grau zero, será denotado por $I_{0}^{r}$, onde $r=g(M)$. Chamaremos de aplicações planar básicas as aplicações associada ao grafo $I_{0}^{r}$.

A Figura 5 ilustra duas aplicações estáveis planares, a primeira com grafo $I_{0}^{1}$ e a segunda grafo $I_{0}^{r}$.
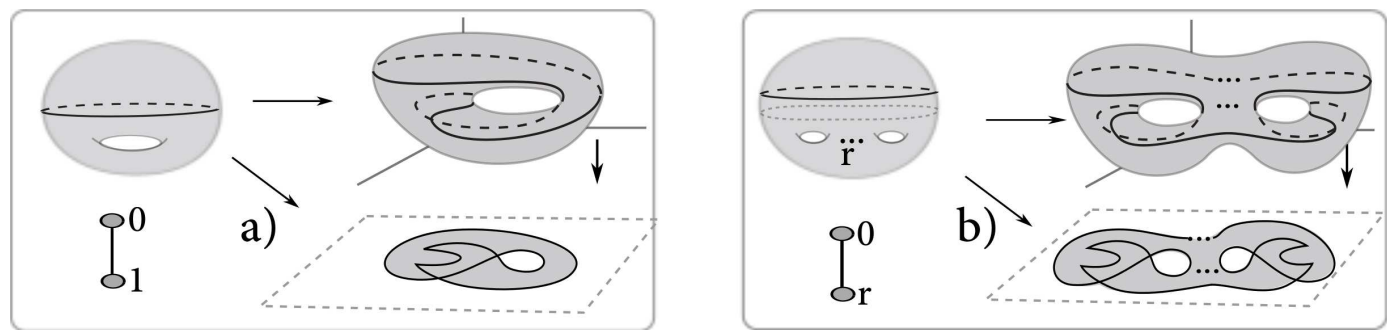

Figura 5: Aplicações planares de grafos com característica $(2,1, T)$.

Se $A$ é uma árvore com $C(A)=(V, V-1,0)$ realizada pela aplicação estável $k: S^{2} \longrightarrow S^{2}$, então $A$ com $C(A)=(V, V-1, T)$, onde $T>0$, t ambém pode realizada por uma aplicação estável $h: Z \longrightarrow \mathbb{R}^{2}$, onde $Z$ é uma superfície fechada e orientada com gênero $T$, da seguinte forma: em cada vértice $v$ de $A$, basta escolher uma aresta $a$ conectada a $v$ e fazer uma soma horizontal entre a aplicação $h$, sobre a curva correspondente a aresta $a$, e aplicação básica associada ao grafo $I_{0}^{t}$, ilustrada na Figura $5 \mathrm{~b}$ ), obtendo assim a aplicação $h$ que realiza $A$. Isto prova o próximo resultado.

Lema 4.2. Todo árvore $A$ com $C(A)=(V, V-1, T)$ pode ser realizada por uma aplicação $g: M \longrightarrow \mathbb{R}^{2}$.

Dado o grafo $G$ com $C(G)=(V, E, T)$, retirando uma aresta de cada ciclo de $G$, obtemos uma árvore $A$ com $C(A)=(V, V-1, T)$. Pelo Lema 4.2 existe uma aplicação estável $g: M \longrightarrow \mathbb{R}^{2}$ que realiza $A$. Por cirurgia horizontal, podemos realizar as arestas do complementar de $A$ em $G$, 
desta forma, obtên-se uma aplicação estável $h: W \longrightarrow \mathbb{R}^{2}$ que realiza $G$, onde o gênero de $W$ pode ser dado por $g(W)=g(M)+\beta_{1}(G)$. Assim temos o resultado geral para grafos associados às aplicações estáveis de superfícies fechadas e orientadas no plano.

Teorema 4.3. Todo grafo bipartido $G$ com $C(G)=(V, E, T)$ pode ser realizado por uma aplicação $f: M \longrightarrow \mathbb{R}^{2}$.

Antes de ver quais grafos com pesos nos vétices podem ser realizados, veremos como realizar um grafo com peso em no máximo uma aresta.

O grafo com única aresta correspondente a uma aplicação estável com grau $d$, será denotado por $I_{d}^{r}$, onde $r=g(M)-d g(N)$. Chamaremos de aplicações básicas com grau $d$ as aplicações associadas ao grafo $I_{d}^{r}$.

A Figura 6 ilustra duas aplicações estáveis sobre a superfície $N$ com gênero $n$, a primeira com grau um e grafo $I_{1}^{0}$, a segunda com grau $d$ e grafo $I_{d}^{0}$.
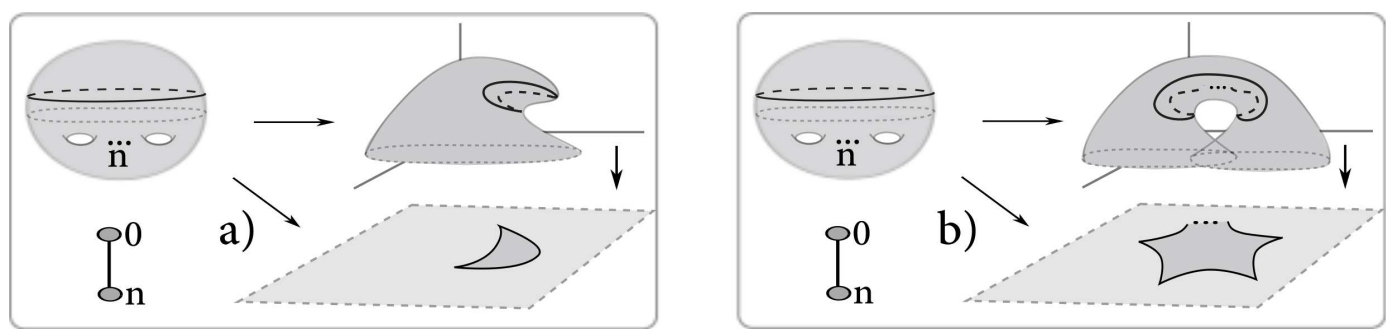

Figura 6: Aplicações com grau $d$ de grafos com característica $(2,1, T)$.

Lema 4.4. Toda árvore com $C=(2,1, T)$ pode ser realizada por uma aplicação $f: M \longrightarrow N$, com grau $d$, onde a superfície $N$ tem gênero $n \geq 0$ e $M$ tem gênero $T=d n+r$, para algum $r \geq 0$.

Demonstração. Para $d=0$, se $T=0$ a aplicação $f$ com única curva singular pode ser a projeção trivial da 2-esfera no plano. Para $T>0, f$ pode ser como as aplicação básica com grafo $I_{0}^{r}$, Figura 5 a) e b). Aplicações com grau $d \neq 0$ e única curva singular pode ser obtida como soma das aplicações básicas com grafo $I_{d}^{0}$, Figura 6 a) e b) e $I_{0}^{r}$.

O próximo resultado generaliza todos os resultados de grafos para aplicações estáveis entre superfícies fechadas e orientadas.

Teorema 4.5. (Teorema de realização) Todo grafo bipartido $G$ com $C(G)=(V, E, T)$ pode ser realizado por uma aplicação estável, sobre uma superfície fechada e orientada $N$ com gênero $n$, com grau no máximo igual a $d=T / n$.

Demonstração. Primeiro, lembramos que toda aplicação $f$ de uma superfície $M$ no plano pode ser considerada (por um mergulho da imagem de $f$ sobre $N$ ) como uma aplicação de grau zero entre duas superfícies $M$ e $N$.

Pelo Teorema 4.3, podemos afirmar que todo grafo bipartido com pesos inteiros positivos nos vértices está associado a alguma aplicação estável $\phi: M \longrightarrow N$ com grau $d=0$.

Veremos como realizar um grafo dado por uma aplicação $\psi \rightarrow N$ com grau máximo. Dado um grafo $G$ com $C(G)=(V, E, T)$, denotamos os pesos nos vértices de $G$ por $t_{1}, \cdots, t_{V}$ e por $r_{i}=t_{i}-d_{i} n$, onde $r_{i}<n$ e $d_{i} \geq 0$. Pelo Teorema 4.3 existe uma aplicação estável $f: S \longrightarrow N$ com grau zero que realiza o grafo $G \operatorname{com} C(G)=(V, E, R)$, onde $R=\sum_{i=1}^{V} r_{i}$.

Fazendo uma cirurgia horizontal entre a aplicação $\phi$ e a aplicação básica com grafo $I_{d_{i}}^{0}$ para cada vértice $v_{i}$ com $d_{i}>0$, obtemos a aplicação $\psi$ com grau $d=\sum_{i=1}^{V} d_{i}$, que realiza o grafo $G$ com $C(G)=(V, E, T)$. 
A Figura 7 ilustra um esquema que pode simplificar bem a ideia, dos resultados apresentados aqui, de como realizar um grafo com pesos nos vértices por uma aplicação sobre uma superfície $n$.

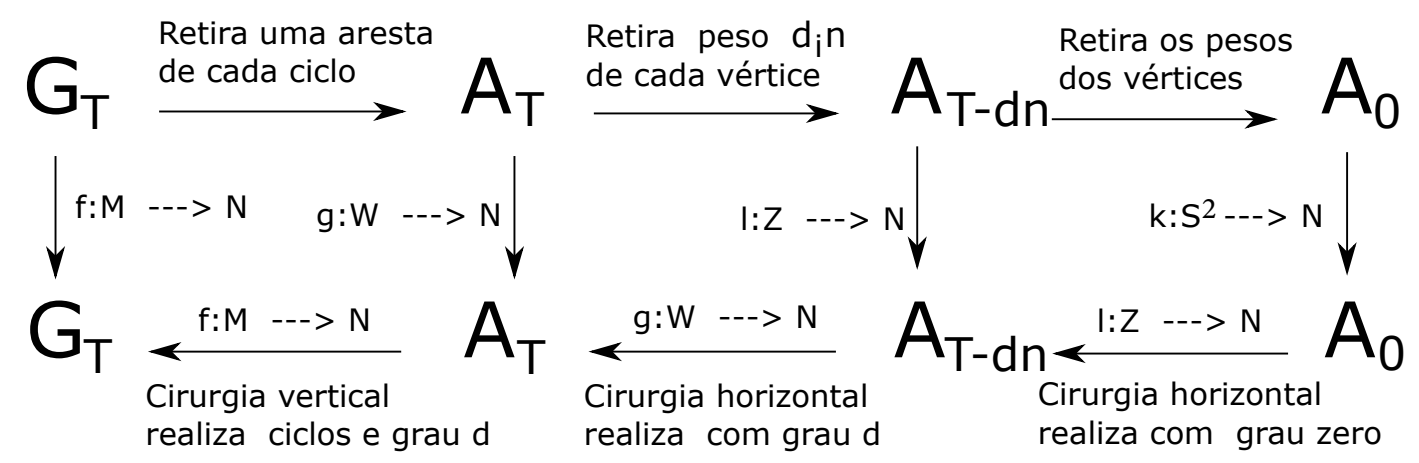

Figura 7: Esquema de realização de grafos com pesos.

\section{Referências}

[1] D. Hacon, C. Mendes de Jesus and M. C. Romero Fuster, Topological invariants of stable maps from a surface to the plane from a global viewpoint. Proceedings of the 6th Workshop on Real and Complex Singularities. Lecture Notes in Pure and Applied Mathematics, 232, Marcel and Dekker, 227-235, 2003.

[2] D. Hacon, C. Mendes de Jesus and M. C. Romero Fuster, Stable maps from surfaces to the plane with prescribed branching data Topology and Its Appl. 154, 166-175, 2007.

[3] D. Hacon, C. Mendes de Jesus and M. C. Romero Fuster, Graphs of stable maps from closed orientable surfaces to the 2-sphere. J. Singularities 2, 67-80, 2010.

[4] C. Mendes de Jesus, "Invariantes Topológicos de Aplicações Genéricas de Superfícies Compactas Orientáveis no Plano", Tese de Doutorado, PUC-RIO, 2001.

[5] C. Mendes de Jesus, Relationship between cusps and regular set of applications between surfaces, preprint 2013.

[6] C. Mendes de Jesus and M.C. Romero Fuster, Graphs of stable maps from closed surfaces to projective plane, preprint 2014.

[7] C. Mendes de Jesus, S. M. Moraes and M. C. Romero Fuster, Stable Gauss maps from a global viewpoint. Bull. Brazilian Math. Soc. 42(1), 87-103, 2011.

[8] C. Mendes de Jesus, R. Oset Sinha and M. C. Romero Fuster, Global topological invariants of stable maps from 3-manifolds to $R^{3}$, Proceedings of the Steklov Institute of Mathematics, 267 (2009) 205-216.

[9] T. Ohmoto and F. Aicardi, First Order Local Invariants of Apparent Coutours, Topology, 45 (2006) $27-45$.

[10] H. Whitney, On Singularities of Mappings of Euclidean Spaces. I. Mappings of the Plane into the Plane, Ann. of Math. 62 (1955) 374-410. 